\title{
Adquisición y Reconocimiento de Imágenes por Medio de Técnicas de Visión e Inteligencia Artificial
}

\author{
Diana Carolina Ramírez González \\ Ingeniera Electrónica, Universidad Escuela \\ Colombiana de Ingeniería Julio Garavito \\ Bogotá, Colombia. \\ Dianita_ramirezg@hotmail.com \\ Beatriz Gerardino Arévalo \\ Ingeniera Electrónica, Universidad Escuela \\ Colombiana de Ingeniería Julio Garavito \\ Bogotá, Colombia. \\ ingchapatina@hotmail.com \\ Enrique Estupiñán Escalante \\ MSc. Profesor de la Universidad Escuela \\ Colombiana de Ingeniería Julio Garavito \\ enrique.estupinan@escuelaing.edu.co
}

\author{
Guillermo Pulido Sarmiento \\ Ingeniero Electrónico, Universidad Escuela \\ Colombiana de Ingeniería Julio Garavito \\ Bogotá, Colombia. \\ ingepulido@hotmail.com \\ José Manuel Cruz Romero \\ Ingeniero Electrónico, Universidad Escuela \\ Colombiana de Ingeniería Julio Garavito \\ Bogotá, Colombia. \\ zeus098@hotmail.com \\ Sandra Cancino Suárez \\ MSc. Profesor de la Universidad Escuela Colombiana \\ de Ingeniería Julio Garavito \\ sandra.cancino@escuelaing.edu.co
}

Resumen- Este proyecto presenta un sistema que permite reconocer diferentes figuras geométricas, que se encuentran en una banda transportadora. Para el posicionamiento de la banda transportadora se utiliza la técnica de Iógica difusa. El control de posicionamiento presenta un error aceptable debido a los protocolos de comunicaciones usados. El reconocimiento de imágenes se realiza a través de técnicas de Visión Artificial y Redes Neuronales.

El sistema de reconocimiento de imágenes consta de tres etapas: adquisición de imágenes digitales a color, procesamiento de imágenes y extracción de las diferentes características de las imágenes e identificación de la figura geométrica. Este sistema puede diferenciar siete formas geométricas (círculo, cuadrado, triángulo, rectángulo, elipse, rombo, estrella de cinco puntas), doce colores (Rojo, rojo claro, rojo oscuro, azul, azul claro, azul oscuro, azul lila, verde, verde claro, verde oscuro, amarillo, amarillo quemado) y tres tamaños (grande, mediano, pequeño). El tratamiento de las imágenes de las figuras requiere técnicas diversas para la identificación de forma como: métrica y diferencia entre ejes principales. Estas características de las imágenes son usadas en la etapa de identificación de las figuras por medio de las técnicas clásicas de visión artificial y a su vez conforma los vectores de entrada a las redes neuronales.

Con los resultados obtenidos se comparan las técnicas de visión artificial y redes neuronales, con el fin de determinar qué herramienta presenta un mejor desempeño en el problema planteado.

Palabras clave- Backpropagation, Filtro Robberts, Filtro Mediana, Filtro Alfa Trimmed, Lógica Difusa, Procesamien- to de Imágenes, Red Neuronal, Controlador Difuso (FLC), Pixeles,

Abstract-This project presents a system that recognizes different figures that are in a conveyor belt. For the conveyor positioning fuzzy logic control is used. The positioning control presents an acceptable error due to the communications protocol used. The techniques used for image recognition are machine vision and neural networks as well.

The image recognition system the system is formed by three stages: image acquisition, image processing and characteristics extraction and geometric form identification. This system differentiate seven geometric shapes (Circle, Square, Triangle, Rectangle, Ellipse, Rhombus, five-pointed Star), twelve different colours (Red, Light Red, Dark Red, Blue, Light Blue, Dark Blue, Lila Blue, Green, Light Green, Dark Green, Yellow, Dark Yellow) and three sizes (Large, Medium, Small). Several techniques are used for different form classification such us: metric and major length axes difference. These image characteristics are used in the geometric identification forms stage by machine vision techniques and these are also the input vectors for the Neuronal Networks.

The results in the image recognition stage are used to compare Machine Vision and Neuronal Network techniques to stablish which one has a better performance for this problem set up.

Keywords-Backpropagation, Robberts Filter, Median Filter, Alfa trimmed Filter, Fuzzy Logic, Image Processing, Neural Network, fuzzy logic controller (FLC), Pixels. 


\section{INTRODUCCIÓN}

El proyecto realiza el reconocimiento de figuras geométricas sobre una banda transportadora por medio de técnicas de visión e inteligencia artificiales. El control de posición de la banda se realiza mediante un controlador de lógica difusa, el cual centra la imagen bajo la cámara y permite la adquisición de la imagen de la figura que se desea clasificar; la clasificación de figuras geométricas en la banda transportadora requiere primero de un procesamiento que incluye el filtrado del ruido, la segmentación por umbralización y la aplicación de filtros de realce de bordes como el filtro Robberts [1], esto para mejorar la imagen y facilitar la extracción de las características útiles para un análisis posterior. Luego, se usan técnicas tradicionales de procesamiento de visión artificial y de computación flexible, específicamente, redes neuronales para clasificar las figuras geométricas de acuerdo a los criterios de forma, tamaño y color definidos previamente. Finalmente, se hace un análisis y una comparación de las dos técnicas implementadas.

El sistema de control de posición se muestra en la Fig. 1, la referencia está dada por la cantidad de píxeles negros esperados en la imagen tomada y el sistema de adquisición extrae la cantidad de píxeles negros en la imagen adquirida, el error es la entrada al controlador difuso (FLC) [5] y la salida es el porcentaje de PWM. El controlador difuso (FLC) está implementado en un microcontrolador PIC 16F877A como se muestra en la Fig. 2 , este microcontrolador se encarga de recibir la referencia del computador y genera el PMW que va a alimentar el motor DC que mueve la banda.

FIG. 1. SISTEMA DE CONTROL DE POSICIÓN DE LA BANDA TRANSPORTADORA CON UN CONTROLADOR DIFUSO (FLC)

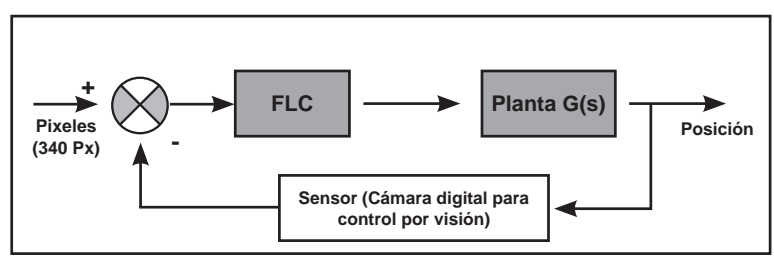

Fuente: Autores del proyecto
FIG. 2. HARDWARE QUE CONFORMA EL SISTEMA DE CONTROL DE POSICIÓN DE LA BANDA TRANSPORTADORA

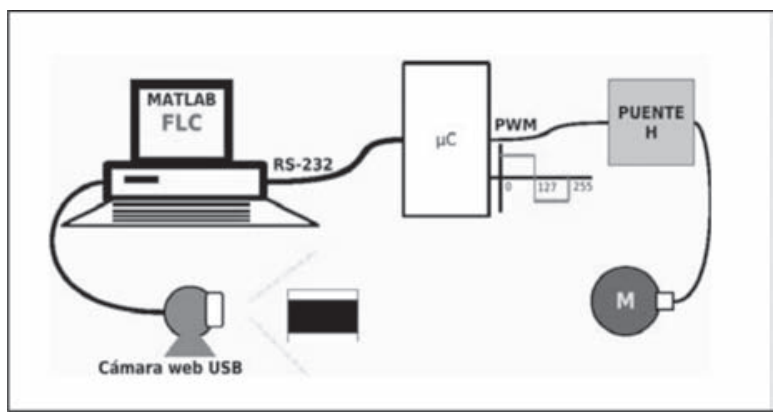

Fuente: Autores del proyecto.

Para evitar que el ambiente afecte significativamente el sistema se adiciona una lámpara de luz blanca y se aísla la iluminación externa con una estructura de color negro que rodea la banda transportadora.

Para la clasificación de las figuras se definieron las siguientes categorías:

1) Forma: cuadrado, rectángulo, triángulo, círculo, elipse, rombo, estrella de cinco puntas.

2) Tamaño: pequeño (área figura $<19 \mathrm{~cm}^{2}$ ), mediano $\left(19 \mathrm{~cm}^{2} \leq\right.$ área figura $\left.\leq 38 \mathrm{~cm}^{2}\right)$, grande (38 $\mathrm{cm}^{2} \leq$ área figura $\leq 56 \mathrm{~cm}^{2}$ ).

3) Color: 12 colores (rojo, rojo claro, rojo oscuro, azul, azul claro, azul oscuro, azul lila, verde, verde claro, verde oscuro, amarillo, amarillo quemado) definidos por el modelo RGB.

\section{DESARROLLO}

\section{A. Construcción del Sistema}

La primera etapa es la construcción física de la banda transportadora que está compuesta por: un motor eléctrico (16 Voltios DC), una cámara Web digital con una resolución de $480 \times 640$ píxeles, un soporte para la cámara digital, rodillos, un acople mecánico motor-rodillo, soporte para los rodillos, la tela con la que se forma la banda y un computador personal. 
En el proceso de la adecuación de la planta física se definen los parámetros como la altura en la que se encuentra la cámara de la banda que es de $35 \mathrm{~cm}$, el área útil para reconocimiento es de $56 \mathrm{~cm}^{2}$ donde se ubican las figuras geométricas, el material de la banda es una tela semielástica de color negro, la cual permite una fricción adecuada con los rodillos de la estructura y un contraste alto en la imagen.

\section{B. Sistema de Control Difuso}

Para realizar el control de posición del motor DC, se utiliza como elemento sensor la misma cámara Web usada para el reconocimiento de figuras, la cual registra periódicamente el movimiento de la banda. Para simplificar la tarea se agregan unas líneas de color blanco uniformemente separadas a lo ancho de toda la banda; las líneas establecen el área donde se posiciona la figura analizada y permiten encontrar la distancia a la que se encuentra la banda de la posición central del foco de la cámara, es decir, la imagen centrada tiene las dos líneas blancas en cada uno de sus extremos como puede verse en la Fig. 3. La banda se mueve lentamente y la cámara entrega la información al computador que realiza el cálculo del error de posición.

FIG. 3. IMAGEN CENTRADA ENTRE DOS LÍNEAS BLANCAS DE LA BANDA TRANSPORTADORA

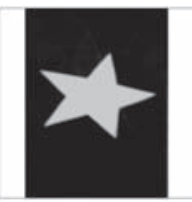

Fuente: Autores del proyecto.

La cámara Web adquiere una imagen a color con una resolución de 480 filas por 640 columnas, la cual es umbralizada con el objetivo de obtener una imagen binaria (blanco y negro). Para realizar el control por lógica difusa, se establece un área de trabajo sobre la imagen binaria de 340 píxeles que corresponde al área de color negro. Cuando la cámara detecta los 340 píxeles de color negro entre las dos franjas blancas, el motor debe parar.

La Fig. 4 presenta una imagen en donde el posicionamiento de la banda tiene un error de posición cero, por tanto, el motor se detiene.

FIG. 4. IMAGEN QUE GENERA UN ERROR DE POSICIÓN CERO EN EL CONTROLADOR DE LA BANDA TRANSPORTADORA

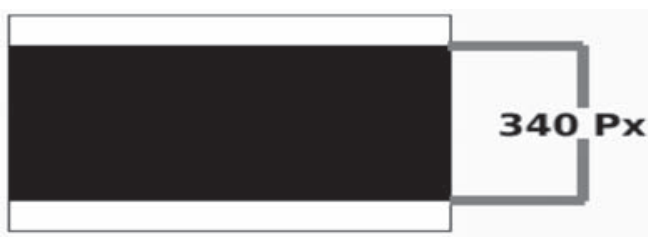

Fuente: Autores del proyecto.

En la Fig. 5 se observan unas imágenes de la banda, en donde su error es distinto de cero, obsérvese que en estos casos existe un proceso de conteo de la cantidad de píxeles de color negro (Y) que hay después de que se detecta la primera línea blanca y basándose en esta información se obtiene el error de posición para usarlo como parámetro de entrada al controlador (Ecuación 1).

FIG. 5. DOS EJEMPLOS CON UN ERROR DE POSICIÓN DIFERENTE DE CERO DE LA BANDA TRANSPORTADORA

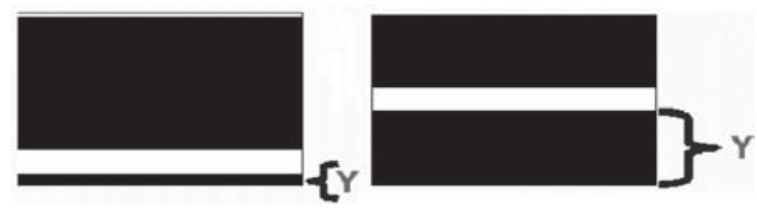

Fuente: Autores del proyecto.

Ecuación 1. ERROR DE POSICIÓN DEL CONTROLADOR DE LA BANDA TRANSPORTADORA

$$
\% \text { error de posición }=\frac{(340-Y) * 100}{340}
$$

\section{1) Implementación del Controlador Difuso}

En la implementación del controlador difuso se tiene como entrada el porcentaje de error y como salida una señal de PWM, como se observa en la Fig. 6 [6]. 
FIG. 6. BLOQUE DE IMPLEMENTACIÓN N DEL CONTROLADOR DIFUSO

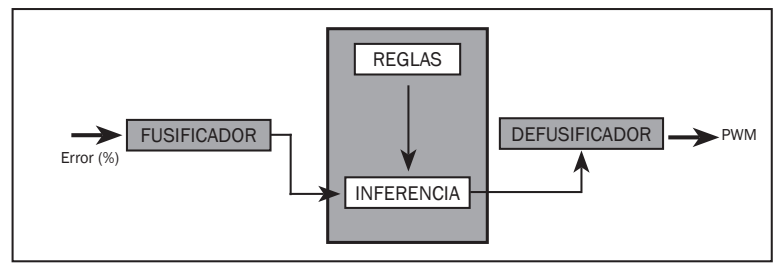

Fuente: [6]. Autores del proyecto

\section{2) Definición de los Conjuntos Difusos}

\section{a. Conjuntos de Entrada}

Para la entrada al controlador se tienen los siguientes valores lingüísticos de la variable del porcentaje de error: Cero, Muy poco, Poco, Medio y Mucho. El universo de discurso está en el rango de $0 \%$ al 100\%, como muestra la Fig. 7 [11].

FIG. 7. CONJUNTOS DE ENTRADA AL FLC

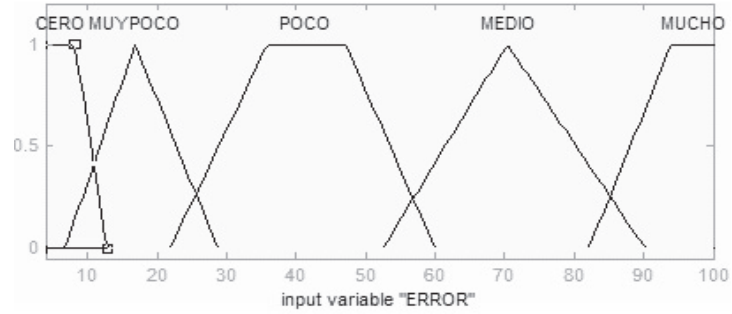

Fuente: Autores del proyecto (Matlab Fuzzy Logic Toolb ox)

\section{b. Conjuntos de Salida}

Para los conjuntos de salida que establecen el valor de PWM que se aplica al motor, se tiene un universo de discurso de 0 a 127, donde cero corresponde a la velocidad más alta que alcanza el motor y 127 cuando el motor detiene la banda transportadora. Las variables lingüísticas definidas para la salida son: Rápido, Despacio, Lento y Quieto, como puede observarse en la Fig. 8 [11].

FIG. 8. CONJUNTOS DE SALIDA DEL FLC

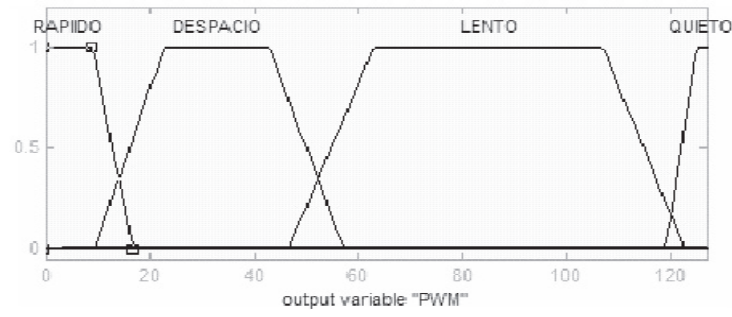

Fuente: Autores del proyecto (Matlab Fuzzy Logic Toolbox)

\section{3) Definición de Reglas}

Se definieron las siguientes variables: la variable de entrada es el error, es decir, la diferencia entre la posición actual y la referencia, son cinco los conjuntos de entrada; y la variable de salida es la velocidad del motor, son 4 los conjuntos de salida. A cada conjunto de entrada le corresponde un conjunto de salida y las reglas se definen:

1. If (error is cero) then (pwm is quieto) (1).

2. If (error is poco) then (pwm is despacio) (1).

3. If (error is muy poco) then (pwm is lento) (1).

4. If (error is mucho) then (pwm is rápido) (1).

5. If (error is medio) then (pwm is despacio) (1).

\section{4) Salida del Defusificador (Técnica centroide)}

Para el cálculo de la salida se utiliza la técnica del centroide para mapear los datos. La Fig. 9 muestra dos ejemplos gráficos de la salida del defusificador [11].

FIG. 9. EJEMPLO DE LA SALIDA DEL DEFUSIFICADOR PARA EL CONTROL DE LA BANDA TRANSPORTADORA

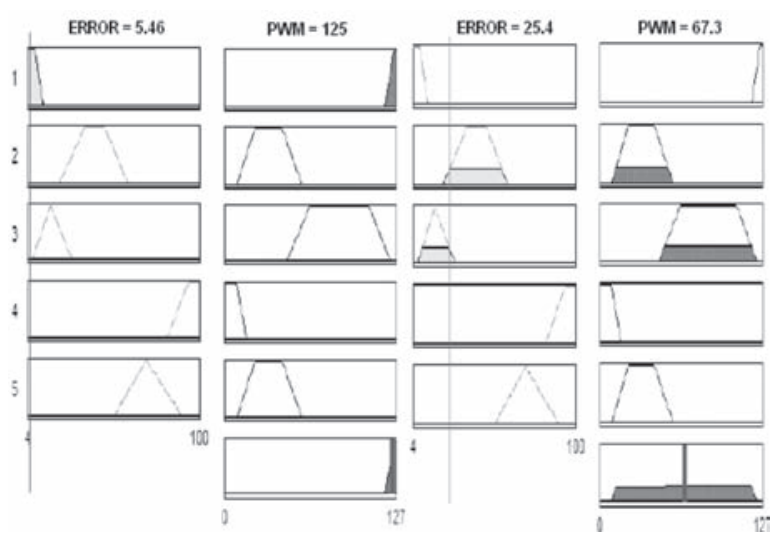

Fuente: Autores del proyecto (Matlab Fuzzy Logic Toolbox)

Para su implementación se generaron tablas con valores de error igualmente espaciados cada 2 seg para obtener el valor correspondiente de PWM. Esta tabla fue almacenada en la posición de memoria del PIC. 


\section{Sistema de Visión Artificial}

\section{1) Adquisición de la Imagen}

Al posicionar de manera correcta la banda transportadora, se procede a capturar la imagen digital de la figura que se va a clasificar. Esta imagen es cargada en el computador en formato de color RGB con una resolución espacial de 480X640 píxeles.

\section{2) Procesamiento de la Imagen y Extracción de} las Características de las Figuras

\section{a) Procesamiento de la Imagen}

Uno de los objetivos de esta etapa es eliminar el ruido que introduce la cámara digital (determinado experimentalmente como ruido gaussiano), para corregirlo se aplica un filtro de mediana a las tres capas de color de la imagen (rojo, azul, verde); pero se observa que el ruido es más fuerte en la capa roja por lo que se decide usar en esta componente un filtro de tipo alfa-trimmed [3]. El siguiente paso es hacer un remuestreo espacial [2] de la imagen obtenida en el paso anterior, que consiste en eliminar 70 píxeles en la parte superior e inferior para eliminar las líneas blancas, obteniendo una imagen como se muestra en la Fig. 10.

FIG. 10. IMAGEN CON REMUESTREO

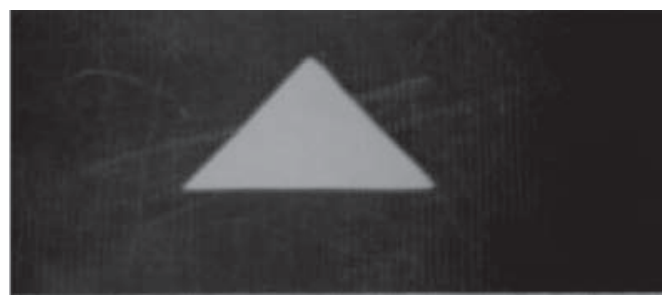

Fuente: Autores del proyecto

A continuación, se realiza la binarización de la imagen que facilita la segmentación de las figuras como puede verse en la Fig. 11. Luego se aplica un filtro de Robberts el cual realza los bordes de las figuras basado en el cálculo del gradiente entre niveles de grises de píxeles vecinos [1], se obtiene así una imagen que destaca el perímetro del objeto como se muestra en la Fig. 11.
FIG. 11. IMAGEN BINARIZADA E IMAGEN CON FILTRO DE ROBBERTS
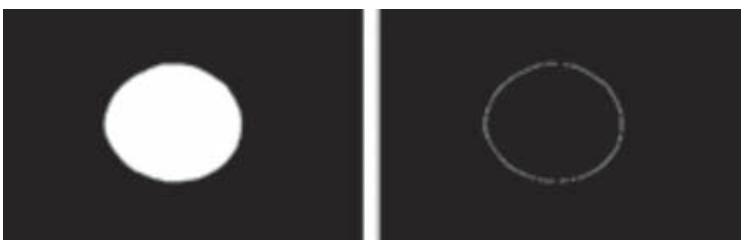

Fuente: Autores del proyecto.

\section{b) Extracción de Características de las Figuras}

La extracción de las características de las figuras permite clasificar la forma, el tamaño y el color de la figura.

\section{- Extracción de las características de Forma de la figura}

Para la extracción de forma se utiliza la imagen binarizada que destaca el área de la figura y la imagen luego del filtro Robberts que destaca el perímetro de la figura (ver Fig. 11.), se realiza un cálculo numérico del área y del perímetro por conteo de píxeles blancos. Posteriormente, con la métrica [3] de las diferentes figuras geométricas y otros atributos como la diferencia entre las longitudes de los ejes principales se realiza la clasificación de forma. Esta distancia es útil para poder diferenciar entre figuras como cuadrado de rectángulo y círculo de elipse ya que su métrica es similar. Por ejemplo: en la Fig. 12. se muestra el caso de un círculo y una elipse, el círculo tiene sus ejes principales iguales, por tanto, la diferencia de sus longitudes de ejes es cero, pero en el caso de la elipse la diferencia de sus longitudes de ejes es distinta a cero.

FIG. 12. LONGITUDES DE LOS EJES DE LA FIGURA DEL CÍRCULO Y LONGUTUDES DE LOS EJES DE LA FIGURA DE LA ELIPSE
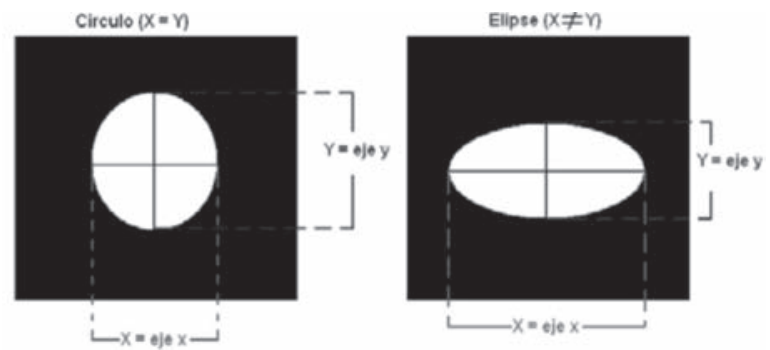

Fuente: Autores del proyecto 


\section{- Extracción de las características de color de la figura}

El modelo RGB asigna un valor de intensidad a cada píxel que oscila entre 0 (negro) y 255 (blanco) para cada una de las componentes RGB de una imagen en color. Por ejemplo, una gama del color rojo podría tener un valor $\mathrm{R}$ de 146 , un valor $\mathrm{G}$ de 20 y un valor $\mathrm{B}$ de 50 . El rojo más puro que se puede conseguir es el R: 255, G: 0 y B: 0 . Cuando los valores de las tres componentes son idénticos, se obtiene un matiz gris. Si el valor de todas las componentes es de 255 , el resultado será blanco y será negro si todas las componentes tienen un valor 0 [1].

Para la identificación de color se utilizan los histogramas de cada componente de la imagen remuestreada la cual puede verse en la Fig. 10. Con los histogramas se obtiene el valor pico de cada componente de color, conforma un nuevo vector de color, así se puede clasificar el tono más representativo de la figura.

\section{- Extracción de las características del tamaño de la figura}

Para la extracción de las características del tamaño solamente se usa la imagen binarizada, con la cual se calcula el área de la figura por medio del conteo de píxeles blancos.

\section{3) Identificación de la Figura}

\section{a) Clasificación de Forma de la Figura}

Para la clasificación de forma se utiliza los valores extraídos del área y del perímetro, con los cuales se realiza una relación denominada métrica [3] para determinar la forma que tiene la figura que se analiza. Sin embargo, en algunos casos mencionados anteriormente, se necesita el valor de la diferencia entre las longitudes de los ejes principales de la figura para identificar su forma geométrica.

\section{b) Clasificación del Color de la Figura}

La identificación de color de la figura se realiza con los valores extraídos de los histogramas de las tres componentes: rojo, verde y azul (RGB). El nuevo vector de color es comparado con valores de referencia generados por un observador humano el cual determinó los rangos para cada uno de los siguientes colores: rojo, rojo claro, rojo oscuro, verde, verde claro, verde oscuro, azul, azul claro, azul oscuro, azul lila, amarillo, amarillo quemado.

\section{c) Clasificación del Tamaño de la Figura.}

Para la clasificación de tamaño se extrae de la imagen de la figura el valor del área y se compara con los siguientes rangos predefinidos y así establecer el tamaño de la figura: figura pequeña de 6000 hasta 17000 píxeles, figura mediana de 17000 hasta 30000 píxeles, figura grande de 30000 hasta 50000 píxeles.

\section{Sistema de Computación Flexible}

El método de computación flexible utilizado es la técnica de Redes Neuronales. Se usa la red neuronal Backpropagation, ya que este tipo de red neuronal permite un aprendizaje supervisado en el cual se determina la salida deseada para cada conjunto de entrada y el ajuste de pesos de las conexiones entre neuronas se realiza desde la salida hasta la entrada [4].

Esta red se ajusta a la definición del problema, ya que se tienen las figuras referentes a las que se asimilan las figuras obtenidas por la adquisición de imágenes. Las figuras referentes son analizadas y se determinan las características de área normalizada, perímetro normalizado, la diferencia de ejes normalizada, métrica y color, que conforman los vectores de los parámetros de entrada a cada una de las redes implementadas.

Loa parámetros de entrada se normalizan con el fin de minimizar el tiempo de entrenamiento.

\section{1) Desarrollo de la red para la clasificación de forma de la figura}

La red de clasificación de forma tiene 3 entradas: el área normalizada con un rango [0 1], el perímetro normalizado con un rango [0 1] y la diferencia de ejes normalizada con un rango [0 1]. Estas entradas son los mismos valores usados en las técnicas de visión artificial. 
El número de capas de la red es cinco, una capa de entrada con tres neuronas, tres capas ocultas y una capa de salida con tres neuronas. La función de activación para las primeras cuatro capas es la función tangente sigmoidea [12]. La salida es un vector de valores binarios que permite clasificar la forma así: cuadrado [0 0 0], rectángulo [0 0 1], circulo [0 1 0], Elipse [ [0 1 1], triángulo [1 0 0], rombo [ [ $\left.\begin{array}{llll}1 & 0 & 1\end{array}\right]$ y estrella [ $\left.\begin{array}{llll}1 & 1 & 0\end{array}\right]$.

\section{2) Desarrollo de la red para la clasificación de color de la figura}

La red de clasificación de color tiene 3 entradas: la componente roja con un rango [0 255], la componente azul con un rango [0 255] y la componente verde con un rango [0 255]. Estas entradas son los mismos valores usados en las técnicas de visión artificial.

El número de capas de la red es cuatro, una capa de entrada con tres neuronas, dos capas ocultas y una capa de salida. La función de activación para las primeras tres capas es la función tangente sigmoidea y la capa de salida tiene 5 neuronas de salida lineal [12]. La salida es un número binario que permite identificar el color, los posibles valores de salida que puede tener la red neuronal son 12 colores, la clasificación de color en la salida con el vector binario es: azules [ $\left[\begin{array}{lllll}0 & 0 & 0 & 0 & 0\end{array}\right]$ al $\left[\begin{array}{lll}0 & 0\end{array}\right.$ $\left.\begin{array}{llll}0 & 1 & 1\end{array}\right]$, rojos [ [ $\left.\begin{array}{lllll}0 & 0 & 1 & 0 & 0\end{array}\right]$ al [ [ $\left.\begin{array}{lllll}0 & 0 & 1 & 1 & 1\end{array}\right]$, verdes [0 $\left.\begin{array}{llll}1 & 0 & 0 & 0\end{array}\right]$ al [ [ [ $\left.\begin{array}{lllll}0 & 0 & 1 & 1\end{array}\right]$ y amarillos [ $\left.\begin{array}{llllll}0 & 1 & 1 & 0 & 0\end{array}\right]$ al [0 $1101]$.

\section{3) Desarrollo de la red para la clasificación de tamaño de la figura}

La red de tamaño tiene una sola entrada que es el área normalizada con un rango de [0 100] píxeles, la cual tiene los mismos valores que se utilizan en las técnicas de visión artificial.

El número total de capas de la red es cuatro, una capa de entrada que tiene tres neuronas, dos capas ocultas y una capa de salida lineal que tiene dos neuronas. La función de activación para las dos capas ocultas es la función tangente sigmoidea [12].
Esta salida es un vector binario, el cual tiene 3 valores diferentes que se establecieron en la red antes del entrenamiento como posibles salidas, cada valor tiene una clasificación: pequeño: [0 0], mediano:[0 1] y grande: [1 0].

\section{PRUEBAS Y RESULTADOS}

\section{A. Control de Posición de la Banda Transportadora}

El control de posición por lógica difusa, tiene un error máximo del $5 \%$ en el posicionamiento debido a los retardos naturales en el protocolo de comunicaciones usado, ya que hay que regular la velocidad a la que se transmiten los datos al microcontrolador. Para este sistema se implementa un retardo de 2 segundos cada vez que se envía un dato al microcontrolador para no congestionar el puerto.

\section{B. Identificación de Forma, Color y Tamaño de las Figuras}

Para realizar las pruebas se escogió una cantidad definida de catorce figuras, con cada figura se realizan 3 pruebas, las figuras están enumeradas y descritas en la Tabla I.

TABLA I

DESCRIPCIÓN DE LAS FIGURAS UTILIZADAS PARA LAS PRUEBAS DEL SISTEMA

\begin{tabular}{|c|c|c|c|}
\hline No. Figura & Color & Tamaño & Forma \\
\hline 1 & Verde oscuro & Pequeño & Cuadrado \\
\hline 2 & Azul claro & Grande & Estrella \\
\hline 3 & Rojo & Pequeño & Elipse \\
\hline 4 & Rojo & Mediano & Círculo \\
\hline 5 & $\begin{array}{c}\text { Rojo pasión } \\
\text { (oscuro) }\end{array}$ & Mediano & Cuadrado \\
\hline 6 & $\begin{array}{c}\text { Amarillo } \\
\text { quemado }\end{array}$ & Mediano & Estrella \\
\hline 7 & Azul lila & Pequeño & Círculo \\
\hline 8 & Azul claro & Pequeño & Triángulo \\
\hline 9 & Verde claro & Pequeño & Rectángulo \\
\hline 10 & Verde claro & Pequeño & Triángulo \\
\hline 11 & Rojo suave & Grande & Cuadrado \\
\hline 12 & Verde & Pequeño & Estrella \\
\hline 13 & Amarillo claro & Pequeño & Cuadrado \\
\hline 14 & $\begin{array}{c}\text { Amarillo } \\
\text { quemado }\end{array}$ & Mediano & Círculo \\
\hline
\end{tabular}

Fuente: Autores del proyecto 


\section{Clasificación por técnicas de Visión Artificial}

La identificación de forma presenta problemas, ya que no sólo se usa la métrica de las figuras sino que fue necesario implementar algunas, la distancia entre ejes para poder diferenciar figuras, como se mencionó anteriormente. Este método de la diferencia de ejes en visión artificial no funciona de una manera óptima, ya que su error es aproximadamente del $65 \%$, posiblemente es por los rangos que se establecieron en la diferencia de longitud de ejes. En la identificación de color se producen cambios notables con sólo alterar la iluminación sobre la banda. En la Tabla II se puede observar los resultados obtenidos de las diferentes pruebas por técnicas de visión artificial. El porcentaje de aciertos en forma con métrica y diferencia de longitud de ejes es de $50 \%$ de acierto, así mismo el color tiene un acierto de $78.57 \%$ y la clasificación del tamaño un $64.28 \%$ de acierto.

TABLA II

RESULTADOS DE LAS PRUEBAS HECHAS AL SISTEMA DE CLASIFICACIÓN POR TÉCNICAS DE VISIÓN ARTIFICIAL

\begin{tabular}{|c|c|c|c|}
\hline $\begin{array}{c}\text { No. } \\
\text { Figura }\end{array}$ & $\begin{array}{c}\text { Acierto en } \\
\text { Color }\end{array}$ & $\begin{array}{c}\text { Acierto en } \\
\text { tamaño }\end{array}$ & $\begin{array}{c}\text { Acierto en } \\
\text { forma }\end{array}$ \\
\hline 1 & $\mathrm{Si}$ & $\mathrm{No}$ & $\mathrm{No}$ \\
\hline 2 & $\mathrm{Si}$ & $\mathrm{No}$ & $\mathrm{Si}$ \\
\hline 3 & $\mathrm{No}$ & $\mathrm{Si}$ & $\mathrm{No}$ \\
\hline 4 & $\mathrm{Si}$ & $\mathrm{No}$ & $\mathrm{No}$ \\
\hline 5 & $\mathrm{No}$ & $\mathrm{Si}$ & $\mathrm{Si}$ \\
\hline 6 & $\mathrm{Si}$ & $\mathrm{No}$ & $\mathrm{Si}$ \\
\hline 7 & $\mathrm{Si}$ & $\mathrm{Si}$ & $\mathrm{Si}$ \\
\hline 8 & $\mathrm{Si}$ & $\mathrm{Si}$ & $\mathrm{Si}$ \\
\hline 9 & $\mathrm{Si}$ & $\mathrm{Si}$ & $\mathrm{No}$ \\
\hline 10 & $\mathrm{Si}$ & $\mathrm{Si}$ & $\mathrm{Si}$ \\
\hline 11 & $\mathrm{Si}$ & $\mathrm{Si}$ & $\mathrm{No}$ \\
\hline 12 & $\mathrm{Si}$ & $\mathrm{Si}$ & $\mathrm{Si}$ \\
\hline 13 & $\mathrm{No}$ & $\mathrm{Si}$ & $\mathrm{No}$ \\
\hline 14 & $\mathrm{Si}$ & $\mathrm{No}$ & No \\
\hline
\end{tabular}

Fuente: Autores del proyecto

\section{Clasificación por Redes Neuronales}

La clasificación por redes neuronales de color, tamaño y forma, se muestran en la Tabla III donde se puede observar los resultados de las pruebas realizadas por medio de redes neuronales con las figuras seleccionadas. El porcentaje de aciertos de la red neuronal de la clasificación de forma tiene un acierto de $64.28 \%$, así mismo la red neuronal de color tiene un $71.43 \%$ de acierto y la clasificación de tamaño tiene un acierto de 57.14\%.
Inicialmente se implementa el entrenamiento por mínimo gradiente, éste presenta un largo tiempo de entrenamiento con poca eficiencia en los casos de clasificación de forma de las figuras y clasificación de colores de las figuras, por tanto, se decidió realizar un segundo entrenamiento por mínima longitud, el cual gasta menos tiempo de entrenamiento y una mayor eficiencia.

TABLA III

RESULTADOS DE LAS PRUEBAS HECHAS AL SISTEMA DE CLASIFICACIÓN POR REDES NEURONALES

\begin{tabular}{|c|c|c|c|}
\hline $\begin{array}{c}\text { No. } \\
\text { Figura }\end{array}$ & $\begin{array}{c}\text { Acierto } \\
\text { en Color }\end{array}$ & $\begin{array}{c}\text { Acierto } \\
\text { en tama- } \\
\text { ño }\end{array}$ & $\begin{array}{c}\text { Acierto en } \\
\text { forma }\end{array}$ \\
\hline 1 & $\mathrm{Si}$ & $\mathrm{Si}$ & $\mathrm{No}$ \\
\hline 2 & $\mathrm{Si}$ & $\mathrm{No}$ & $\mathrm{Si}$ \\
\hline 3 & $\mathrm{Si}$ & $\mathrm{Si}$ & $\mathrm{No}$ \\
\hline 4 & $\mathrm{Si}$ & $\mathrm{No}$ & $\mathrm{No}$ \\
\hline 5 & $\mathrm{Si}$ & $\mathrm{No}$ & $\mathrm{Si}$ \\
\hline 6 & $\mathrm{Si}$ & $\mathrm{No}$ & $\mathrm{Si}$ \\
\hline 7 & $\mathrm{No}$ & $\mathrm{Si}$ & $\mathrm{No}$ \\
\hline 8 & $\mathrm{No}$ & $\mathrm{Si}$ & $\mathrm{Si}$ \\
\hline 9 & $\mathrm{No}$ & $\mathrm{Si}$ & $\mathrm{Si}$ \\
\hline 10 & $\mathrm{Si}$ & $\mathrm{Si}$ & $\mathrm{Si}$ \\
\hline 11 & $\mathrm{Si}$ & $\mathrm{No}$ & $\mathrm{Si}$ \\
\hline 12 & $\mathrm{Si}$ & $\mathrm{Si}$ & $\mathrm{Si}$ \\
\hline 13 & $\mathrm{No}$ & $\mathrm{Si}$ & $\mathrm{Si}$ \\
\hline 14 & $\mathrm{Si}$ & $\mathrm{No}$ & $\mathrm{No}$ \\
\hline
\end{tabular}

Fuente: Autores del proyecto

La Fig. 13. muestra la interfaz gráfica del sistema implementado, en donde se incluyen imágenes de la Figura de la prueba 5 mencionada en las tablas: Tabla I., Tabla II., Tabla III.; histogramas y resultados de las técnicas de visión artificial y redes neuronales.

FIG. 13. INTERFAZ GRÁFICA DEL SISTEMA IMPLEMENTADO QUE OBSERVA EL USUARIO

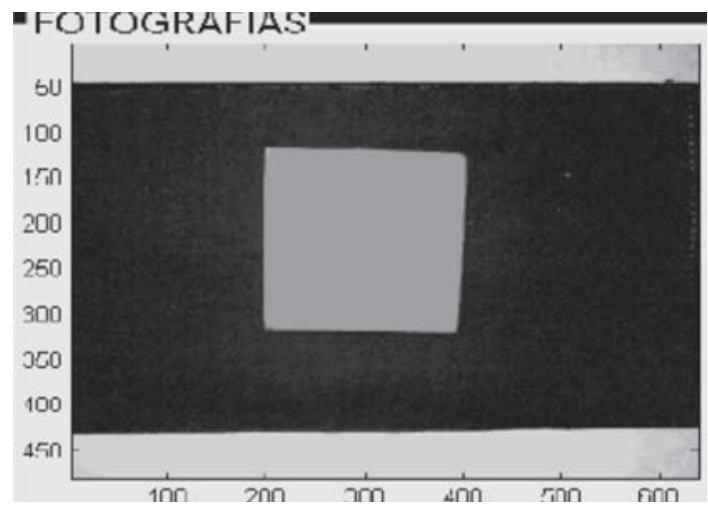



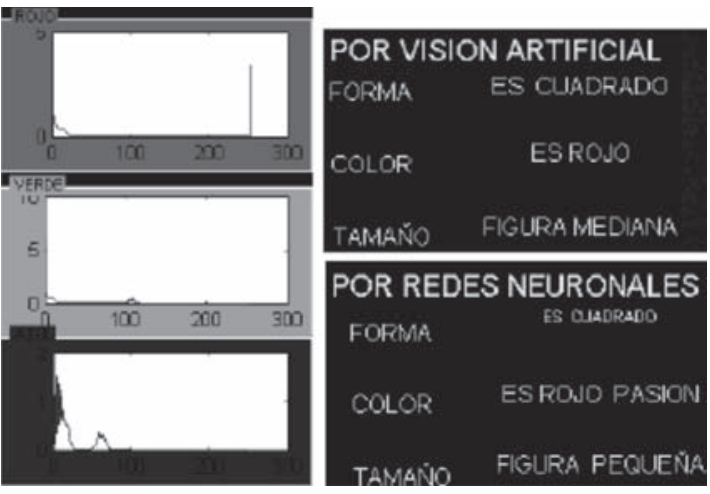

Fuente: Autores del proyecto.

\section{CONCLUSIONES}

Con base en los resultados para la identificación de forma, se puede concluir que la técnica de redes neuronales es más exacta con un $64.28 \%$ de acierto, que por medio de técnicas de visión artificial, la cual obtuvo un $50 \%$ de acierto.

Para la identificación de color los porcentajes muestran que la técnica de visión artificial es más exacta con un $78.57 \%$ de acierto que la técnica de redes neuronales con un $71.43 \%$ de acierto, sin embargo, dada la poca diferencia se requiere realizar más pruebas.

La técnica de redes neuronales es un poco menos exacta para la identificación de tamaño, ya que presenta un $57.14 \%$ de asertividad contra la técnica de visión artificial que presenta un porcentaje de $64.28 \%$ de acierto.

Dado que las técnicas de visión artificial se requieren para el procesamiento y la extracción de las características de la imagen digital, utilizadas en ambas técnicas de clasificación, y la diferencia en la capacidad de acierto es pequeña, no se puede concluir cuál de las dos técnicas es más eficiente para la clasificación de atributos de las figuras seleccionadas para este estudio. Por otra parte, la técnica de redes neuronales requiere del trabajo adicional de entrenamiento y ajuste haciéndola menos atractiva para el problema estudiado.

\section{REFERENCIAS}

[1] R. González, P. Wintz., Digital Image Processing, 3a. edición, Prentice Hall.

[2] M. Sonka, V. Hlvac, R. Boyle, Image Processing, Analysis, and Machine Vision, Brooks/Cole Publishing Co, 1999.

[3] R. González, Woods, and Eddins, Digital Image Processing Using Matlab, 2nd Ed, Prentice Hall.

[4] Fausett L, Fundamentals of Neural Networks: Architecture, Algorithms, and Applications Prentice Hall, 1994.

[5] Klir G, Yuan B, Fuzzy Set Theory. Foundations and Applications, Prentice Hall, 1998.

[6] Pasino K, Yurkovich S, Fuzzy Control, Addison Wesley, 1998.

[7] www.Omega.ilece.edu.mx:3000/sites/ciencia.htm

[8] www.itba.edu.ar/capis/public_html/roboticacognitiva/REDES-NEURONALES.pdf

[9] http://www.answermath.com/neuralnetworks/tutorial-esp-7-aprendizaje.htm

[10] http://www.monografias.com/trabajos12/redneuro/ redneuro.shtml

[11] http://www.gc.ssr.upm.es/inves/neural/ann2/anntutor.htm

[12] Toolbox, MATLAB, Redes Neuronales.

[13] Toolbox, MATLAB, Logical Fuzzy.

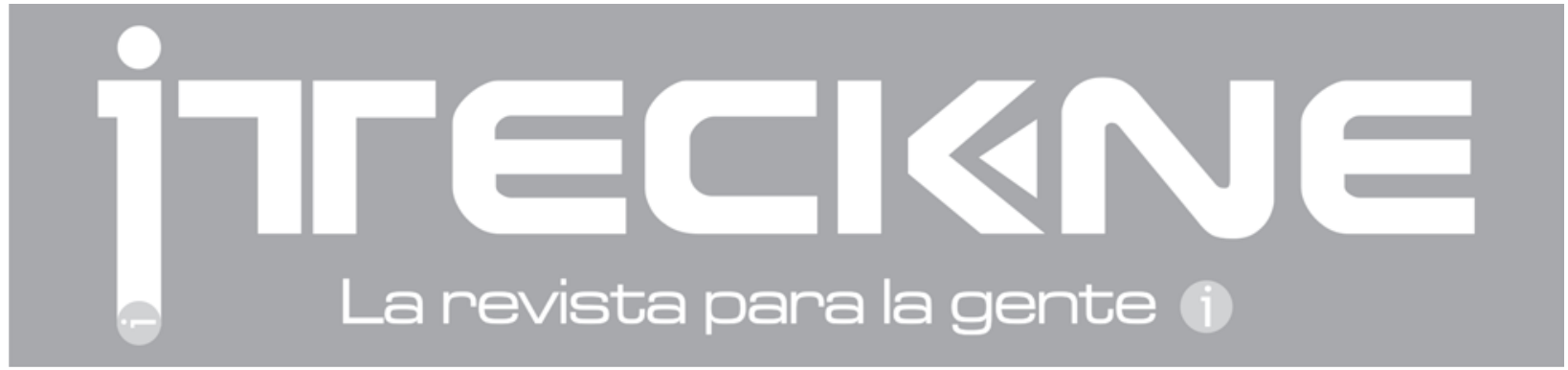

